\title{
Exploring Coupled Extreme Environments via In-situ Transmission Electron Microscopy
}

\author{
Khalid Hattar
}

Sandai National Laboratories, Albuquerque, New Mexico, United States

A large percentage of the extreme applications that materials are exposed to comprise of complex and overlapping stressors. Fundamental mechanisms that govern a materials response to complex extreme environments are often unknown and currently too intertwined to be accurately tested at the atomic-scale. These processes occur at material surfaces, defects sites, grain boundaries, and within site-specific interfaces, where the combination of sample control in atmospheric chemistry, temperature, pressure, radiation conditions, mechanical force, electrical, and magnetic force are needed to produce an operando test. Additionally, current methods in x-ray, optical, infrared, scanning probe, and electron microscopy cannot make full use of combined collection of data signals to enable the event recognition in terms of composition, structure, and reaction on one single sample with known microstructure or composition.

This presentation will explore the state of the field, recent advancements made at Sandia and the future potential to elucidate the fundamental mechanisms associated with a materials' evolution during creation, processing, operation, degradation, and/or failure should come from the atomistic scale. Over the last several decades, advancements in sample preparation, aberration corrected electron optics, and large data processing has rapidly advanced the spatial resolution, and in the breadth of data that can be collected from a TEM sample during an in-situ experiment [1]. In addition, these developments have permitted great advancements in the extent and overlap of the stressor that can be explored (temperature, electrical bias, environments, mechanical loading, etc.). Sandia has developed a unique tool, In-situ Ion Irradiation Transmission Electron Microscope ( $\left.\mathrm{I}^{3} \mathrm{TEM}\right)$, which permits the coupling of some of these extreme environments on a highly modified JEOL 2100. This tools has been utilized to the explore the response of materials to combination of thermal, mechanical, and radiation environments simultaneously [2]. This can be done during quantitative mechanical loading and general structural evolution [2] or during microstructural observation [3]. Recent work has pushed the limits of the coupled in-situ TEM thermal and quantitative mechanical testing to temperates above $2,000{ }^{\circ} \mathrm{C}$ via laser heating [4].

The information that can be obtained and the coupling of these extreme environments currently appears to be limited by the original platform utilized. As such, this presentation will conclude with a thought experiment of an Integrated Transmission Electron Microscope (ITEM) designed for the optimal exploration of a range of complex thermal, mechanical, electrical and radiation environments with atomic spatial resolution and nanosecond temporal resolution with access to all the structural, compositional, and bonding signals emanating from the sample. The development of such a tool would provide direct correlation of atomic-scale structural and compositional mapping, ultrafast imaging, electronic bonding information, and mechanical properties, under controlled extreme environmental conditions is conceivable [5]. 


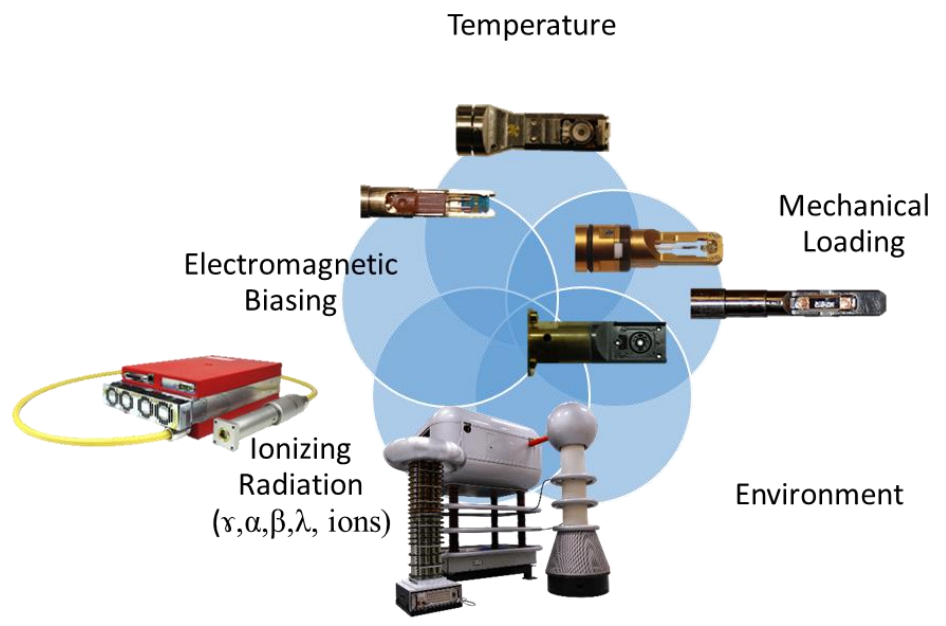

Figure 1. Potential methods to couple extreme environments during an in-situ TEM experiment.

\section{References}

[1] Robertson, Ian M., Christopher A. Schuh, John S. Vetrano, Nigel D. Browning, David P. Field, Dorte Juul Jensen, Michael K. Miller et al. "Towards an integrated materials characterization toolbox." Journal of Materials Research 26, no. 11 (2011): 1341-1383.

[2] Jawaharram, Gowtham Sriram, Patrick M. Price, Christopher M. Barr, Khalid Hattar, Robert S. Averback, and Shen J. Dillon. "High temperature irradiation induced creep in Ag nanopillars measured via in situ transmission electron microscopy." Scripta Materialia 148 (2018): 1-4.

[3] Bufford, Daniel C., Christopher M. Barr, Baoming Wang, Khalid Hattar, and Aman Haque. "Application of In Situ TEM to Investigate Irradiation Creep in Nanocrystalline Zirconium." JOM 71, no. 10 (2019): 3350-3357.

[4] Grosso, Robson, Eliana NS Muccillo, Dereck NF Muche, Gowtham S. Jawaharram, Christopher M. Barr, Anthony M. Monterrosa, Ricardo HR Castro, Khalid Hattar, and Shen J. Dillon. "In situ Transmission Electron Microscopy for Ultrahigh Temperature Mechanical Testing of ZrO2." Nano Letters (2020).

[5] This work was supported by the U.S. Department of Energy, Office of Nuclear Energy under DOE Idaho Operations Office Contract DE-AC07-051D14517 as part of a Nuclear Science User Facilities experiment. This work was performed, in part, at the Center for Integrated Nanotechnologies, an Office of Science User Facility operated for the U.S. Department of Energy (DOE) Office of Science. Sandia National Laboratories is a multimission laboratory managed and operated by National Technology \& Engineering Solutions of Sandia, LLC, a wholly owned subsidiary of Honeywell International, Inc., for the U.S. DOE's National Nuclear Security Administration under contract DE-NA-0003525. The views expressed in the article do not necessarily represent the views of the U.S. DOE or the United States Government. 